American Journal of Applied Sciences 9 (9): 1527-1533, 2012

ISSN 1546-9239

(C) 2012 Science Publication

\title{
Effect of Doping Position on the Active Silicon-on-Insulator Micro-Ring Resonator Based on Free Carrier Injection
}

\author{
Mardiana, B., Sahbuddin Shaari, P. Susthitha Menon, \\ H. Hazura, A.R. Hanim and H. Abdullah \\ Institute of Microengineering and Nanoelectronics (IMEN), \\ Universiti Kebangsaan Malaysia, 43600 UKM Bangi, Selangor, Malaysia
}

\begin{abstract}
Problem statement: Metal interconnects have become significant limitation on the scaling of CMOS technologies in electronics integrated circuit. Silicon photonics has offers great potential to overcome this critical bottleneck due to the advantages of optical interconnects. Silicon-based optical micro-ring resonator is promising basic element of future electronic-photonic integrated circuits because of its wide applications on photonic devices such as modulator, switch and sensor. Approach: This study highlights the study of the free carrier injection effect on the active SOI micro-ring resonator. The effect of the free carrier injection on micro-ring resonator is evaluated by varying the $\mathrm{p}^{+}$ and $\mathrm{n}^{+}$doping position. Device performances are predicted using numerical modeling software 2D SILVACO as well as Finite Difference Time Domain (FDTD) simulation software, RSOFT. Results: The results show that the refractive index change increases as the $\mathrm{p}^{+}$and $\mathrm{n}^{+}$doping position become closer to the rib waveguide. A shift in resonant wavelength of around 2 and $3 \mathrm{~nm}$ was is predicted at $0.9 \mathrm{~V}$ drive forward voltage for 0.5 and $1.0 \mu \mathrm{m}$ gap distance between $\mathrm{p}^{+}$and $\mathrm{n}^{+}$doping regions and the sidewall of the rib waveguide. It is also shown that 10 and $9.2 \mathrm{~dB}$ maximum change of the output response obtained through the output of the transmission spectrum of the device with gap 0.5 and 1.0 $\mu \mathrm{m}$. Conclusion: The closer distance between $\mathrm{p}^{+}$and $\mathrm{n}^{+}$doping regions and the rib waveguide has optimal shift of resonance wavelength and better extinction ratio of transmission spectrum.
\end{abstract}

Key words: Free carrier injection, p-i-n diode structure, Micro-ring resonator, Silicon-on-Insulator (SOI)

\section{INTRODUCTION}

Photonic Integrated Circuits (PIC) is expected to grow rapidly in terms of its density and owing to these more concise optical components are required (Bo, 2011; Thylen et al., 2006). It has been proven lately that the Silicon-On-Insulator (SOI)-based optical micro-ring resonator is one of the most important components for more complex photonic integrated circuits due to its compactness (Xu et al., 2005). The importance of the micro-ring resonator is highlighted in various applications such as filtering, combining, processing, sensing and conversion of the optical signal (Barrios and Lipson, 2004; Xu et al., 2007; Liu et al., 2007; Li et al., 2007).

SOI technology has profound advantages due to its high refractive index in the course to obtain a sound optical confinement (Soref and Bennet, 1987). Based on this reason, SOI is the right substrate material for the optical micro-ring. Besides that, the low operational cost and the compatibility of the SOI with current microelectronic fabrication technique is also another advantageous reason for its usage (Tang and Reed, 1995; Png et al., 2004). SOI also has a unique property where it is transparent in the range of optical telecommunications wavelengths of 1.3 and $1.55 \mu \mathrm{m}$ (Hewitt and Reed, 2000).

There are two types of common structures of optical SOI microring resonator as shown in Fig. 1. The use of different structures depends on the application. Fig. 1a shows the microring structure consisting of a circular waveguide coupled with two straight waveguides while Fig. 1b shows the second structure of microring in which the circular waveguide is coupled to only one straight waveguides. In this study, we have designed the device based on the first structure.

Microring principle begins with the resonant wavelength from the input port of the straight waveguide evanescently coupled to the circular ring waveguide. The coupling condition is determined by the three main factors; microring radius, refractive index and the wavelength of input light.

Corresponding Author: Mardiana, B., Institute of Microengineering and Nanoelectronics (IMEN), Universiti Kebangsaan Malaysia, 43600 UKM Bangi, Selangor, Malaysia 


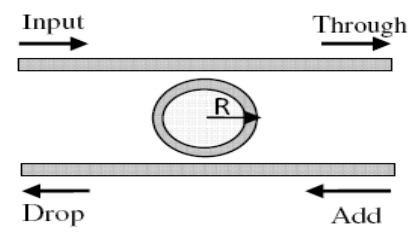

(a)

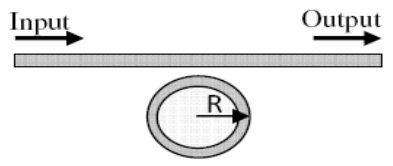

(b)

Fig. 1: Schematic of a micro-ring resonator

The relationship is described in the equation below (Chao and Guo, 2006):

$\mathrm{n}_{\text {eff }} \mathrm{L}=\mathrm{m} \lambda$

Where:

$\mathrm{m}=$ An integer

$\lambda=$ The input wavelength

$\mathrm{n}_{\mathrm{eff}}=$ The effective index of the circular waveguide

$\mathrm{L}=$ The length of the circular waveguide

The coupling coefficients of the couplers are determined by the gap (g) between the straight waveguide and the ring (Chin and Ho, 1998). Theoretically, power transmission outputs at the drop port can be predicted by (Xioa et al, 2007):

$$
\mathrm{T}=\frac{4 \mathrm{x}\left[\frac{\mathrm{FSR}}{4 \pi}\right]^{2}\left[\mathrm{k}^{4}\right]}{\left[\lambda-\lambda_{\mathrm{o}}\right]+\left[\frac{\mathrm{FSR}}{4 \pi}\right]^{2}\left[2 \mathrm{k}^{2}+\mathrm{k}_{\mathrm{p}}{ }^{2}\right]^{2}}
$$

Where:

$\lambda_{\mathrm{o}}=$ The resonant wavelength

$\mathrm{k}^{2}=$ The power coupling

$\mathrm{k}_{\mathrm{p}}^{2}=$ Coefficient between the bus waveguide and the resonator

Free Spectral Range (FSR) is another important observed parameter. From the transmission spectrum, the FSR can be estimated by observing the difference between two consecutive resonant peaks. Wide FSR is demanded in the integrated optics whereby more channels can be accommodated:

$$
\mathrm{FSR}=\frac{\lambda_{o}}{n_{\text {eff }}\left(2 \pi R+2 L_{c}\right)}
$$

Where:

$\mathrm{R}=$ The ring radius

LC $=$ The coupler length. From more channels can be accommodated

All the pre-determined parameter of the micro ring's structure designed will influence the characteristic of the device such as the resonant wavelength. Since the micro-ring's characteristics cannot be modified and controlled, one way to controlling or tune its characteristics is by adding an active element towards the waveguide. The refractive index of the waveguide material is one of the parameters which can be changed and controlled. It is commonly known that silicon-based material does not exhibit linear electro-optic (Pockels effect and the Kerr effect is very weak). Therefore, the refractive index of the silicon can be varied by free carrier dispersion effect and thermal effect (Soref and Bennet, 1987).

In this study we have designed an active SOI ring resonator with $\mathrm{p}$-i-n diode structure integrated on the ring waveguide. The tuning of the refractive index was performed using the free carrier dispersion effect through forward injection free carrier at the p-i-n diode structure along the ring. The changes of the refractive index will influence the resonance condition of the device. The induced real refractive index $(\Delta \mathrm{n})$ at the wavelength of $1.55 \mu \mathrm{m}$ is given by (Reed and Knights, 2004):

$$
\Delta \mathrm{n}=-\left(8 \times 10^{-22} \Delta \mathrm{Ne}+8.5 \times 10^{-18}(\Delta \mathrm{Nh})^{0.8}\right)
$$

Where:

$\Delta \mathrm{Ne}=$ The changes of the free electrons concentrations $\Delta \mathrm{Nh}=$ The changes of the free holes concentrations

Initial studies shows that the effects of free carrier injection on microring resonator's characteristics are influence by the applied drive voltage and doping concentrations. Other than these factors, the doping positions of $\mathrm{p}^{+}$and $\mathrm{n}^{+}$from the waveguide are also important. Different positions will cause different free carrier injection distributions in the active region of the micro-ring (Yong et al., 2012). Here, we systematically study the effects of the free carrier injection by varying the highly doped position $\left(\mathrm{n}^{+}\right.$ and $\mathrm{p}^{+}$) from the rib waveguide. The gap is indicated as B in Fig. 2.

\section{MATERIALS AND METHODS}

Figure 2 shows the cross section of the active region of the SOI micro-ring resonator to be studied in this study. 


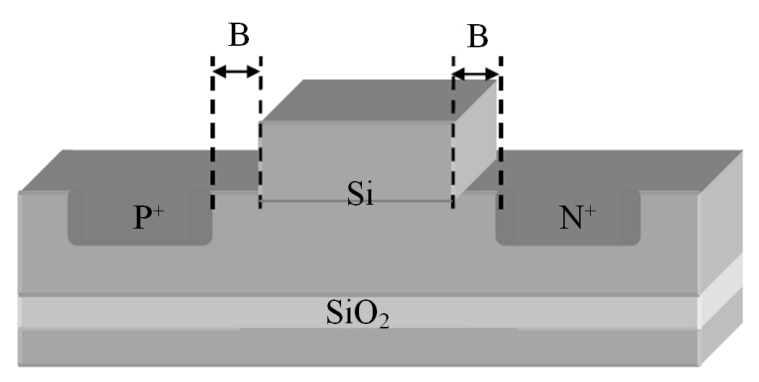

Fig. 2: Cross section of the active region

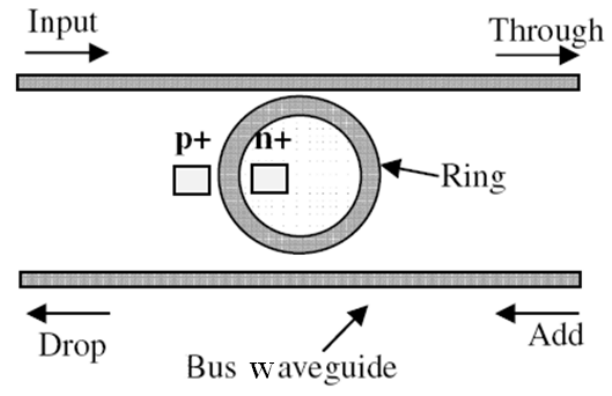

Fig. 3: Schematic of the SOI micro-ring resonator with a $\mathrm{p}-\mathrm{i}-\mathrm{n}$ diode embedded in the ring waveguide

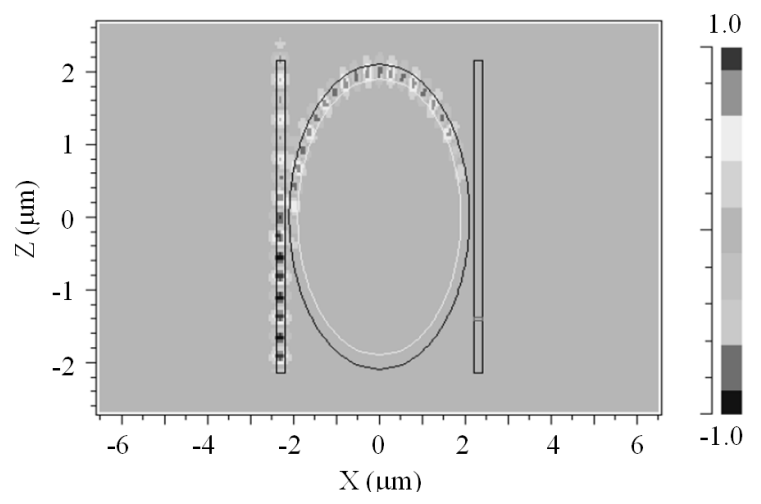

Fig. 4: Simulation image of micro-ring resonator by using R-Soft Sofware

The active region consists of a $\mathrm{p}-\mathrm{i}-\mathrm{n}$ diode structure integrated into the rib waveguide. Standard silicon microelectronics fabrication processes was used to model the device by using the 2D ATHENA module from SILVACO software (Lennie et al., 2009). The active section was formed on the silicon-on-insulator substrate by implanting the $\mathrm{p}-\mathrm{i}-\mathrm{n}$ diode structure at the ring waveguide. The silicon layer is lightly doped with concentration of $1 \times 10^{14} \mathrm{~cm}^{-3}$, whereas both $\mathrm{p}^{+}$and $\mathrm{n}^{+}$ region is implanted with boron and phosphorus. B is a gap distance of the $\mathrm{p}^{+}$and $\mathrm{n}^{+}$doping region position from the sidewall waveguide (Jahanshah et al., 2009).

The whole structure of the micro-ring is illustrated in Fig. 3. The micro-ring has been designed with 200 $\mathrm{nm}$ gap spacing between the bus and the ring waveguide. The rib waveguide has been determined, with the rib height of $580 \mathrm{~nm}$, rib width of $500 \mathrm{~nm}$. The proposed design has been simulated using R-soft software to determine the optical behavior. Fig. 4 shows the simulated image of micro-ring in R-Soft.

\section{RESULTS}

In order to study the electrical characteristics of the active region, the 2D ATLAS module from the SILVACO software was employed. Meanwhile, the resonance characteristics have been analyzed by using the FDTD method in R-Soft software.

The DC electrical simulations have been performed on the active region of the micro-ring resonator. The analyses have been done by applying an external electrical signal to the electrodes. By observing the doping concentrations variations at the waveguide centre $(x=2 \mu \mathrm{m}, \mathrm{y}=3.05 \mu \mathrm{m})$, it enables us to work out the refractive index changes at a specific forward bias voltage. From the calculated electron and hole concentration values, the free carrier induced variation in real refractive index can be obtained by using Eq. 4 .

Fig. 5 shows the refrective index change versus applied voltage for different gap distance between $\mathrm{p}+$ and $n+$ doping region position from the sidewall of rib waveguide. The following graph shows the transmission spectrum of the microring resonator at different drive forward voltages. The transmission spectrum of the device is obtain from Fig. 6 and 7 represent the gap of the 0.5 and $1.0 \mu \mathrm{m}$ respectively. Basically, the simulation of the transmission spectrum by the simulator is done based on Eq. 1 and 2 .

Fig. 8 shows the comparison of resonance wavelength shift for different applied forward voltage and Fig. 9 indicates the correlation between the wavelength shift and the applied drive voltage. The results curve show that the resonance wavelength shifted exponentially with increment of the applied voltage both for the gap of 0.5 and $1.0 \mu \mathrm{m}$. Finally, Fig. 10 shows the transmission spectrum of the micro-ring resonator with different position of the doping regions.The corresponding maximum Free Spectral Range (FSR) of 50 and $55 \mathrm{~nm}$ was obtained for the gap of 0.5 and $1.0 \mu \mathrm{m}$. The FSR of the device can be calculated by Eq. 3 as well. 
Am. J. Applied Sci., 9 (9): 1527-1533, 2012

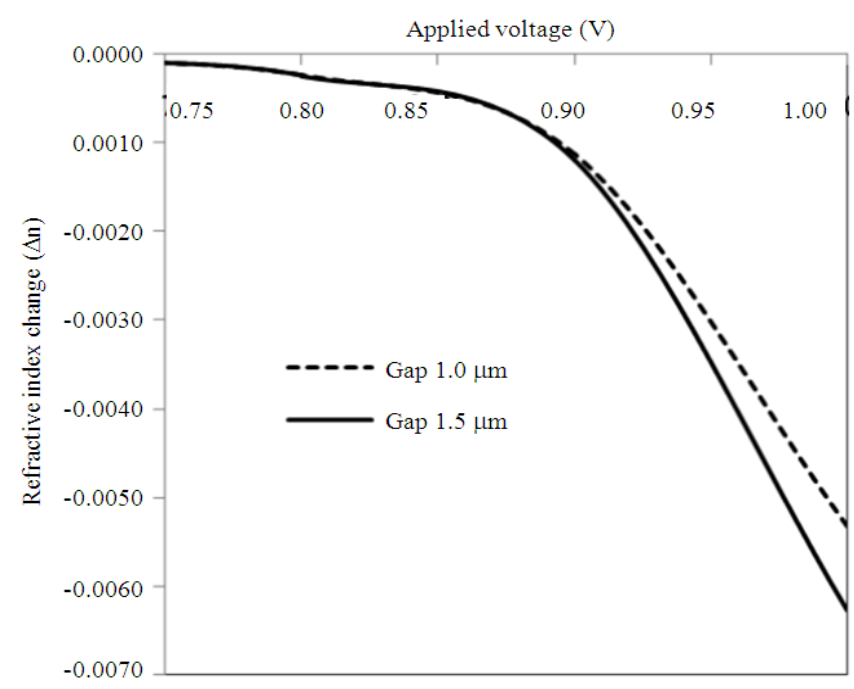

Fig. 5: Refractive index changes with increment of forward applied voltage

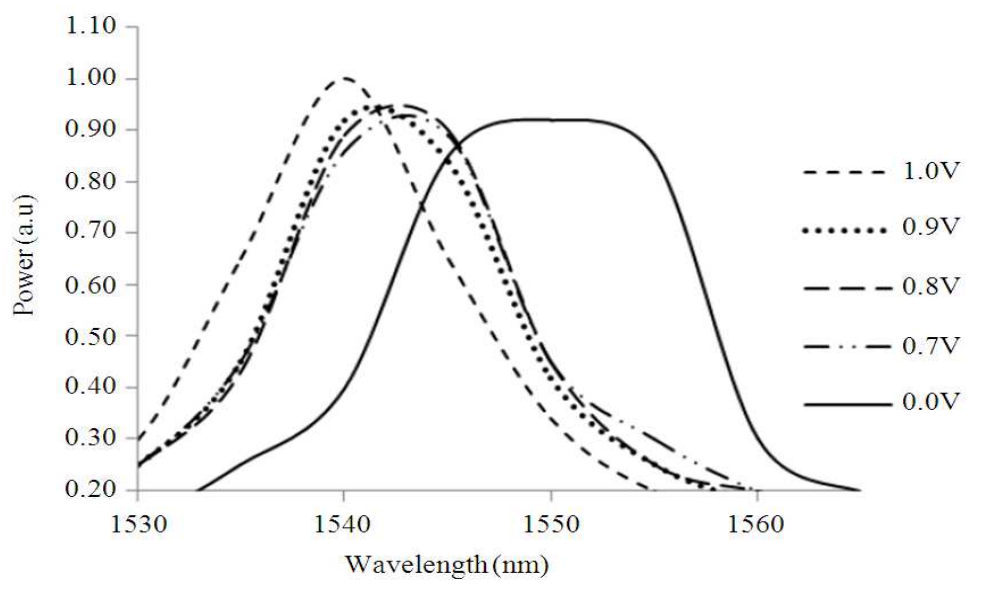

Fig. 6: Transmission spectrum with different applied voltage at gap $0.5 \mu \mathrm{m}$

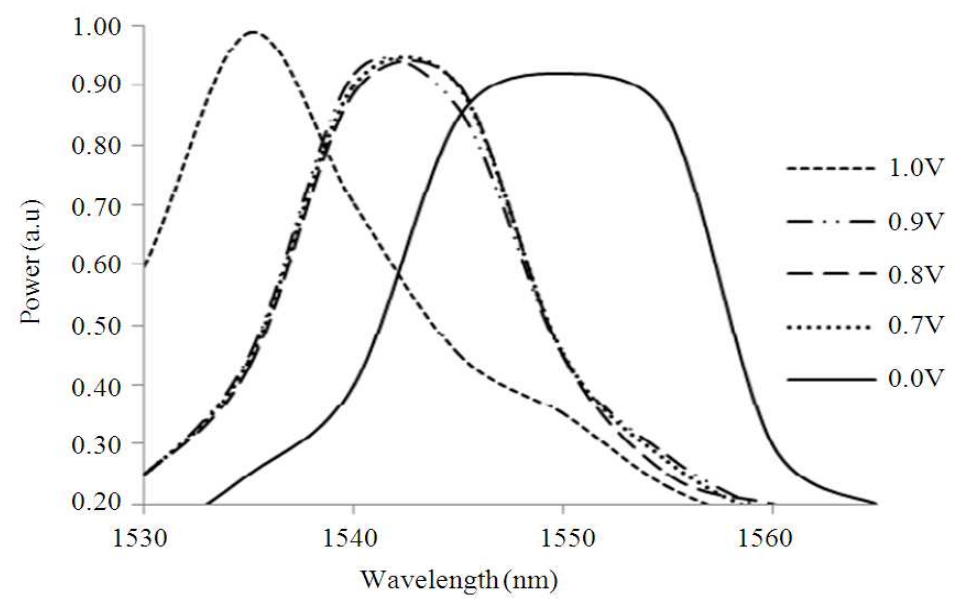

Fig. 7: Transmission spectrum with different applied voltage at gap $1.0 \mu \mathrm{m}$ 1530 
Am. J. Applied Sci., 9 (9): 1527-1533, 2012

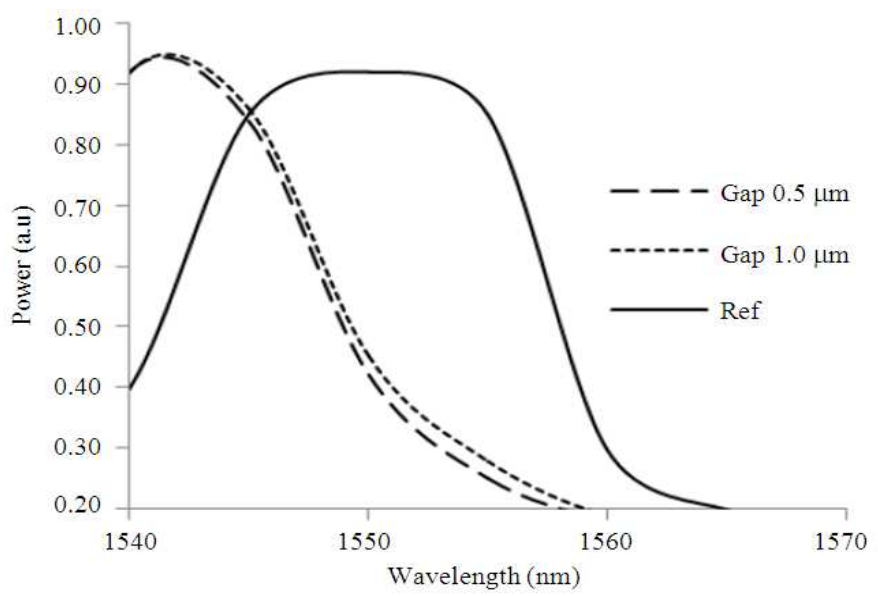

Fig. 8: Resonant wavelength shift for different applied forward voltage

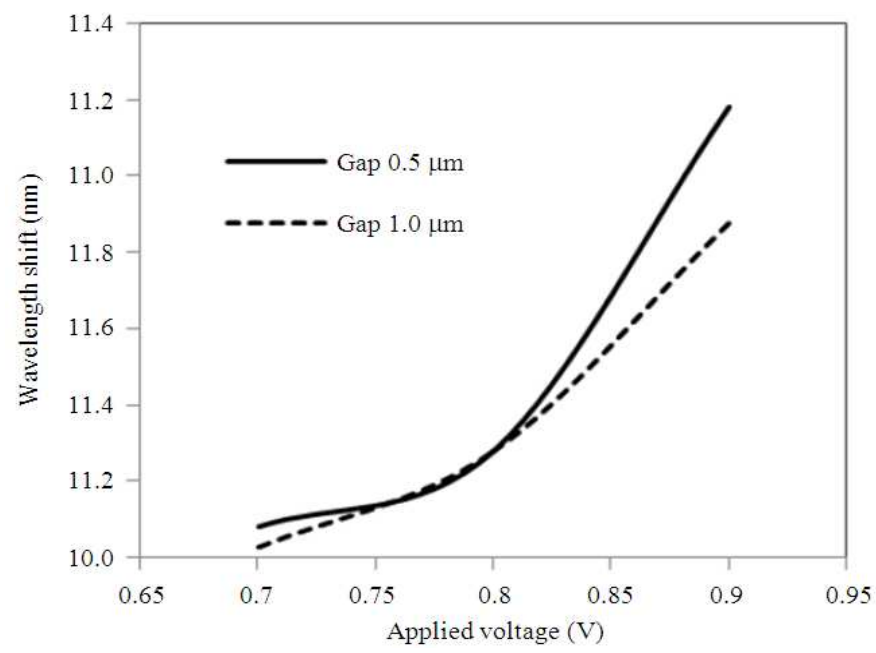

Fig. 9: Summarized of resonant wavelength shift for different applied forward voltage

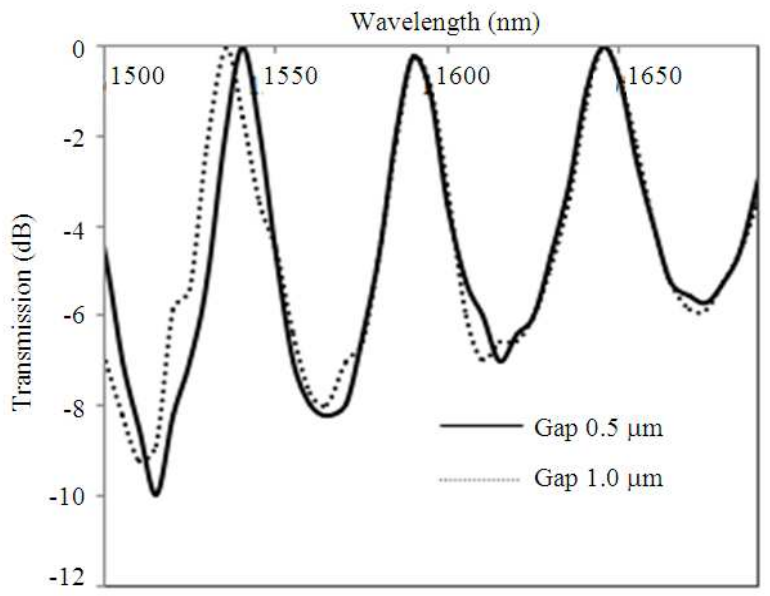

Fig. 10: Transmission spectrum of the proposed device 1531 


\section{DISCUSSION}

Refractive Index Change: The refractive index change increased as the gap of the doping region was brought closer to the rib waveguide sidewall. As an example, at $1 \mathrm{~V}$ drive forward voltage, the refractive index change rose from $5.33 \mathrm{E}-03$ at $1 \mu \mathrm{m}$ gap to $6.28 \mathrm{E}-03$ at $0.5 \mu \mathrm{m}$ gap. The average increment percentage is about $17.8 \%$. This happened due to the reduced distance for the injected free holes and the free electrons to move from the doping region to the optical waveguide.It caused an increment in the free carrier density at the guided region.

Transmission spectrum: By referring to Fig. 6 and Fig. 7, it was proven that the changes in the refractive index were due to the free carrier injection in which it was responsible to the lower-wavelength shift of the resonant wavelength. From the results, the transmission of the light was also increased as more forward voltage has been applied to the device. It was clearly seen that the highest value of applied voltage was responsible for the biggest shift of the resonant wavelength. Obviously, the closer the distance between the doping regions have biggest resonance wavelength shift and high change of output response.

Resonant wavelength shift: The resonance wavelength shifted exponentially with increment of the applied voltage both for the gap of 0.5 and $1.0 \mu \mathrm{m}$. In addition, the peak power of optical light was also improved by $5.4 \%$. It is concluded that by increasing the applied voltage, the resonant wavelength shifts further as expected. Thus it is proven that the effect of the free carrier injection become bigger when the gap between the doping region and the rib waveguide become closer.

Free Spectral Range (FSR): The good FSR results are due to the very small size of the designed micro-ring.

\section{CONCLUSION}

The effects of the free carrier injection on the active SOI optical micro-ring resonator was evaluated by varying the position of the highly doping regions of $\mathrm{p}^{+}$and $\mathrm{n}^{+}$. The refractive index change $(\Delta \mathrm{n})$ increased and the resonant wavelength shifted optimally as the doping positions become closer to the rib waveguide. Understanding of the free carrier injection effect on the device's characteristics is very important for the future photonic interconnection technology. Future work will be focus on the optimization of the microring resonator performances based on the free carrier injection effect.

\section{ACKNOWLEDGMENT}

The researchers would like to thank Universiti Teknikal Malaysia Melaka (UTeM) for the support. The Ministry of Higher Education of Malaysia and Universiti Kebangsaan Malaysia (UKM) are gratefully acknowledged for the grant under Industrial Project2011-015.

\section{REFERENCES}

Lennie, A., H. Abdullah, S. Shaari and K. Sopian, 2009. Fabrication of Single Layer $\mathrm{SiO}_{2}$ and $\mathrm{Si}_{3} \mathrm{~N}_{4}$ as Antireflection Coating on Silicon Solar Cell Using Silvaco Software. Am. J. Applied Sci., 6: 20432049. DOI: 10.3844 /ajassp.2009.2043.2049

Barrios, C.A. and M.Lipson, 2004. Modeling and analysis of high-speed electro-optic modulation in high confinement silicon waveguides using metaloxide-semiconductor configuration. J. Applied Phys., 96: 6008-6015. DOI: 10.1063/1.1814791

Chin, M.K. and S.T. Ho, 1998. Design and modeling of waveguide-coupled single-mode microring resonators. J. Lightwave Technol., 16: 1433-1446. DOI: 10.1109/50.704609

Cung-Yen Chao and L.Jay Guo, 2006. Design and optimization of microring resonators in biochemical sensing applications,: J. Lightwave Technol., 24: 1395-1402. DOI:10.1.1.135.4174

Jahanshah, F., K. Sopian, S. H. Zaidi, M. Y. Othman and N. Amin et al., 2009. Modeling the Effect of P$\mathrm{N}$ Junction Depth on the Output of Planer and Rectangular Textured Solar Cells. Am. J. Applied Sci., 6: 667-671. DOI: 10.3844/ajassp.2009.667.671

Hewitt, P.D. and G.T. Reed, 2000. Improving the response of optical phase modulators in SOI by computer simulation. J. Lightwave Technol., 18: 443-450. DOI: 10.1109/50.827519

Li, C., L. Zhou and A.W. Poon, 2007.Silicon microring carrier-injection-based modulators/switches with tunable extinction ratios and OR-logic switching by using waveguide cross-coupling. J. Optics Express, 15: 5069-5076. DOI: 10.1364/OE.15.005069

Liu, A., L. Liao, D. Rubin, H. Nguyen and B. Ciftcioglu et al., 2007. High-speed optical modulation based on carrier depletion in a silicon waveguide. J. Optics Express, 15: 660- 668. DOI: 10.1364/OE.15.000660

Png, C.E., S.P. Chan, S.T. Lim and G.T. Reed, 2004. Optical phase modulators for $\mathrm{MHz}$ and $\mathrm{GHz}$ modulation in Silicon-On-Insulator (SOI). J. Lightwave Technol., 22: 1573-1582. DOI: 10.1109/JLT.2004.827655 
Reed, G.T. and A.P. Knights, 2004. Silicon Photonics: An Introduction. 1st Edn., John Wiley and Sons, Chichester, ISBN: 9780470870358, pp: 276.

Soref, R. and B. Bennett, 1987. Electrooptical effects in silicon. IEEE J. Quantum Elect., 23: 123-129. DOI: 10.1109/JQE/1987.1073206

Tang, C.K. and G.T. Reed, 1995. Highly efficient optical phase modulator in SOI waveguides. Elect. Letter, 31: 451-452. DOI: 10.1049/el:19950328

Thylen, L., S. He., L. Wosinki and D. Dai, 2006. The Moore's Law for photonic integrated circuits. J. Zhejiang Univ. Sci., 7: 1961-1967. DOI: 10.1631/jzus.2006.A1961

Xioa, S., M.H. Khan, H. Shen and M. Qi, 2007. Modeling and measurement of losses in silicon-oninsulator resonators and bends. J. Optics Express, 15: 10553-10561. DOI: 10.1364/OE.15.010553
Xu, Q., S. Manipatruni, B. Schmidt, J. Shakya and M. Lipson., 2007. 12.5 Gbit/s carrier-injection-based silicon micro-ring silicon modulators. J. Optics Express, 15: 430- 436. DOI: 10.1364/OE.15.000430

Xu. Q., B. Schmidt, S. Pradhan and M. Lipson, 2005. Micrometre-scale silicon electrooptic modulator. Nature, 435: 325-327. DOI: 10.1038/nature03569

Bo, Y., 2011. Effects of Adhesive on Coupling Efficiency of Planar Light Waveguide Packaging. American Journal of Nanotechnology., 1: 68-77. DOI: 10.3844/ajnsp.2010.68.77

Yong, Z., W. Wanjun, S. Haifeng, Y. Jiany and W. Minghua et al., 2012. Influence of doping position on the extinction ratio of Mach-Zehnderinterference based silicon optical modulators. J. Semiconductor, 33: 1-3. DOI: 10.1088/16744926/33/1/014009 